\title{
A Modelica library for Thermal-Runaway Propagation in Lithium-Ion Batteries
}

\author{
Christian Groß Andrey W. Golubkov \\ Virtual Vehicle Research GmbH, Austria, batterysafety@v2c2 . at
}

\begin{abstract}
Based on the Thermal Runaway (TR) experiments conducted in our laboratory a simple method of describing a battery's thermal behaviour was developed. In the approach - which we call simple tracing method the temperature rate measurement from Accelerating Rate Calorimetry (ARC) during a TR experiment is approximated to determine the thermal behaviour of the model. This method was implemented in Modelica using Dymola. Alongside the implementation of the TR model a complete Modelica package with useful models for TR propagation simulation was developed. The package called "BatterySafety" serves as a foundation for further model development to investigate TR propagation and physical counter measures in greater detail. The focus of all the models in the package was efficiency, intelligibility and user-friendliness. With this approach we are able to simulate TR propagation of a complete battery pack.

Keywords: Lithium-Ion Batteries, Battery Safety, Thermal Runaway, Thermal Runaway Propagation
\end{abstract}

\section{Introduction}

\section{List of Acronyms}

- TR - Thermal Runaway

- LIB - Lithium-Ion Battery

- ARC - Accelerating Rate Calorimetry

- SOC - State-Of-Charge

\section{Motivation}

Batteries of electric vehicles are safe at temperatures below $\approx 80^{\circ} \mathrm{C}$. If $\mathrm{Li}$-ion cells reach temperatures above $80^{\circ} \mathrm{C}$ the cells start to degenerate and when they reach a even higher temperatures they may transit into thermal runaway (TR). An over temperature of a cell is a major safety concern and may be caused by failures inside the cell or by the surrounding of the cell. In automotive applications, where energy storage usually does not consist of a single cell but a battery pack of multiple connected cells, TR is an even bigger concern. The battery pack allows for TR propagation, where failure in one cell causes failure in adjacent cells, which can ignite the whole battery pack. Understanding and predicting TR and TR propagation are key to the development of countermeasures.
Feng, Lu, et al. (2016) said, what could not have been said better: "Experimental study on TR propagation is essential, and we may need massive experiments on TR propagation to help design a safe battery pack. [...] However, given that the experimental study on TR propagation within a battery pack costs much time and money, building an easy-to-use, verified abuse model that realistically captures the mechanisms of TR propagation in battery pack is beneficial to find efficient approaches to prevent TR propagation." In that spirit the BatterySafety library was developed, backed by the knowledge our team gathered over the years (Andrey W. Golubkov et al. 2018; Essl, Andrey W. Golubkov, et al. 2020) and will be released under the Modelica License 2.

\section{Paper Structure}

This paper consists of six sections, starting with the introduction in section 1 . In section 2 we are going to look at the mathematical backbone of the library and introduce the simple tracing model. Section 3 then discusses the implementation of the library with a focus on key models. And section 4 showcases the functionality of the library by looking at some example simulations, followed by section 5 and section 6 the discussion and conclusion of the paper.

\section{Simple Tracing Model}

The key element of the BatterySafety library is the TR model around which the library is built. This section aims to educate about our modelling approach, it's benefits and weaknesses. First we lay out the foundation, then we process that into a implementable form and last we talk about how the heating rate data needed for the model is obtained.

\subsection{Deriving the model}

Consider the thermal runaway being a simple chemical reaction

$$
A \rightarrow B
$$

with the concentration $c$ of the educt $A$ going from 1 to 0

$$
c=1 \rightarrow c=0
$$

The chemical reaction happens inside a Li-ion cell with a fixed heat capacity $C_{p}$ and an overall enthalpy change $\Delta H$. The temperature rate of the Li-ion cell during TR depends on the kinetics of the chemical reaction $\dot{c}$ and on the heat 
exchange with the ambient $\alpha_{A} \Delta T$ ( $\alpha_{A}$ is the heat transfer coefficient times the area).

$$
\dot{T}=-\frac{\Delta H}{C_{p}} \dot{c}(T)+\alpha_{A} \Delta T
$$

which is a rough approximation of the first law of thermodynamics. Consider a ideal adiabatic reaction with $\alpha=0$; no heat exchange with the ambient

$$
\dot{T}=-\frac{\Delta H}{C_{p}} \dot{c}(T)
$$

Rewrite and consider the temperature dependence of $\dot{T}$

$$
\dot{c}(T)=-\frac{C_{p}}{\Delta H} \dot{T}(T)
$$

The temperature rate $\dot{T}(T)$ is given by the piecewise linearisation in the rate plot of the experiment, as depicted in Figure 1. In the rate plot $g(T)$ follows the measured temperature between $150^{\circ} \mathrm{C}$ and $300^{\circ} \mathrm{C}$. The measured decrease of the rate at temperatures $T>300^{\circ} \mathrm{C}$ is ignored in $g(T)$, because it is caused by spatial effects (spatial heat flow during the experiment). At $T<150^{\circ} \mathrm{C}$ the self heating could not be detected by the measurement equipment (because it is obscured by the external heating at $>1{ }^{\circ} \mathrm{C} / \mathrm{min}$ ), but it is known from other publications (Feng, Zheng, et al. 2019) that the slope can extrapolated below $150^{\circ} \mathrm{C}$.

During simulation the model traces $\dot{T}(T)$ : it starts with some elevated temperature, extracts the $\dot{T}(T)$ from the rate plot and updates the temperature. In the next time step the model extracts $\dot{T}(T)$ with the updated temperature and so on until all educts are consumed $(c=0)$ then the rate is forced to zero $\dot{T}(c \leq 0)=0$.

$$
\dot{T}(T)= \begin{cases}\text { linear approximation, } & \text { if } c>0 \\ 0, & \text { otherwise }\end{cases}
$$

The overall enthalpy change $\Delta H$ is calculated from the maximal temperature change during the experiment

$$
\Delta H=C_{p}\left(T_{\max }-T_{\text {onset }}\right)
$$

To successfully and efficiently implement the simple tracing model in Modelica, we need to adapt the equations a little. Instead of considering the concentration $c$, we want to think in terms of available energy $E$, which we assume to depend linearly on the $c$

$$
E(T)=\Delta H c(T)
$$

We can now express Equation 5 in terms of energy as well

$$
\dot{E}(T)=-C_{p} \dot{T}(T)
$$

which can be implemented in a straight forward way as Section 3.2 shows.

The simple tracing model has two main disadvantages
- The heat loss to the ambient is not considered, therefore the values of $\dot{T}(T)$ and $\Delta H$ may be underestimated. The actual error depends on the thermal insulation in experiment setup, e.g. the deviation from ideal adiabatic condition.

- The the piecewise linear heating rate $\dot{T}(T)$ gives no additional insight into the chemical reactions system (order of the reaction, number of Arrhenius reactions)

The big advantage of this approach is that no curve fitting is needed.

\subsection{Thermal-Runaway Experiment}

The data we use to generate the temperature rate approximation is gathered first-hand from conducting TR experiments. To prepare the experiment we first attach temperature sensors on the cell's surface and then apply insulating cover. The cell assembly then is put into the sample holder, which is fitted with heating elements. Through springs the sample holder applies a desired force on the cell. Further the sample holder is inserted into the reactor, the reactor sealed pressure tight and flushed with $\mathrm{N}_{2}$ gas. Last the cell is cycled (dis- and then recharged) and charged to desired state-of-charge (SOC). We then conduct the experiment and let the cell transit into TR by chosen method. Usually TR is induced by heating the cell up to the cell-dependent TR onset temperature. The interested reader can find an in-depth explanation on our method and setup in our other publications. We recommend the work of Essl, A. Golubkov, and Fuchs (2020) and Essl, Andrey W. Golubkov, et al. (2020) as a starting point. For the examples in section 4 we have used data from "Cell \#2" in the paper of Essl, A. Golubkov, and Fuchs (2020).

\section{Library Implementation}

The BatterySafety library features too many models to explain them all in detail in this paper. Therefore we decided to just show the implementation of the core models from the bottom up. We are now going to look at how we implemented the TR model and arrived at the cell, module and battery pack level models.

\subsection{Energy Storage}

At the end of section 2 the equations were expressed in terms of energy, because we wanted to captivate this idea in Modelica. The absolute nature of the stored energy proved to be a surprisingly challenging obstacle in implementation. We started by implementing a light green coloured energy connector

Listing 1. Definition of the energy connector

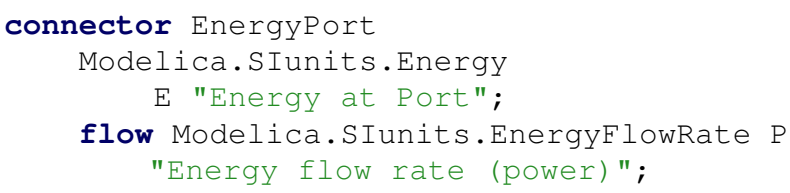




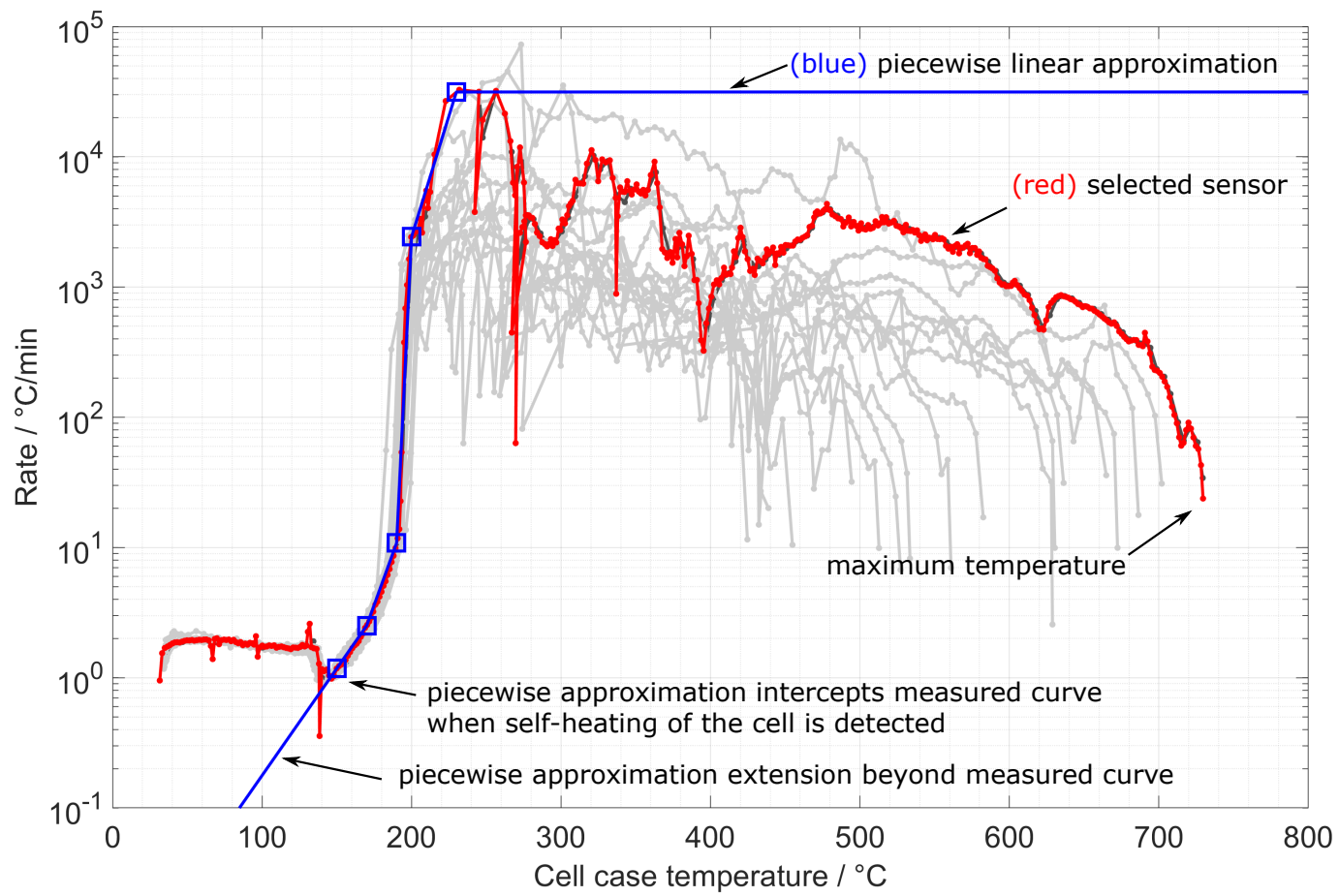

Figure 1. Temperature rate measurements from a single experiment: (red) Selected measurement for modelling, (blue) the linear approximation in logarithmic scale and (grey) measurements from different points on the cell's surface.

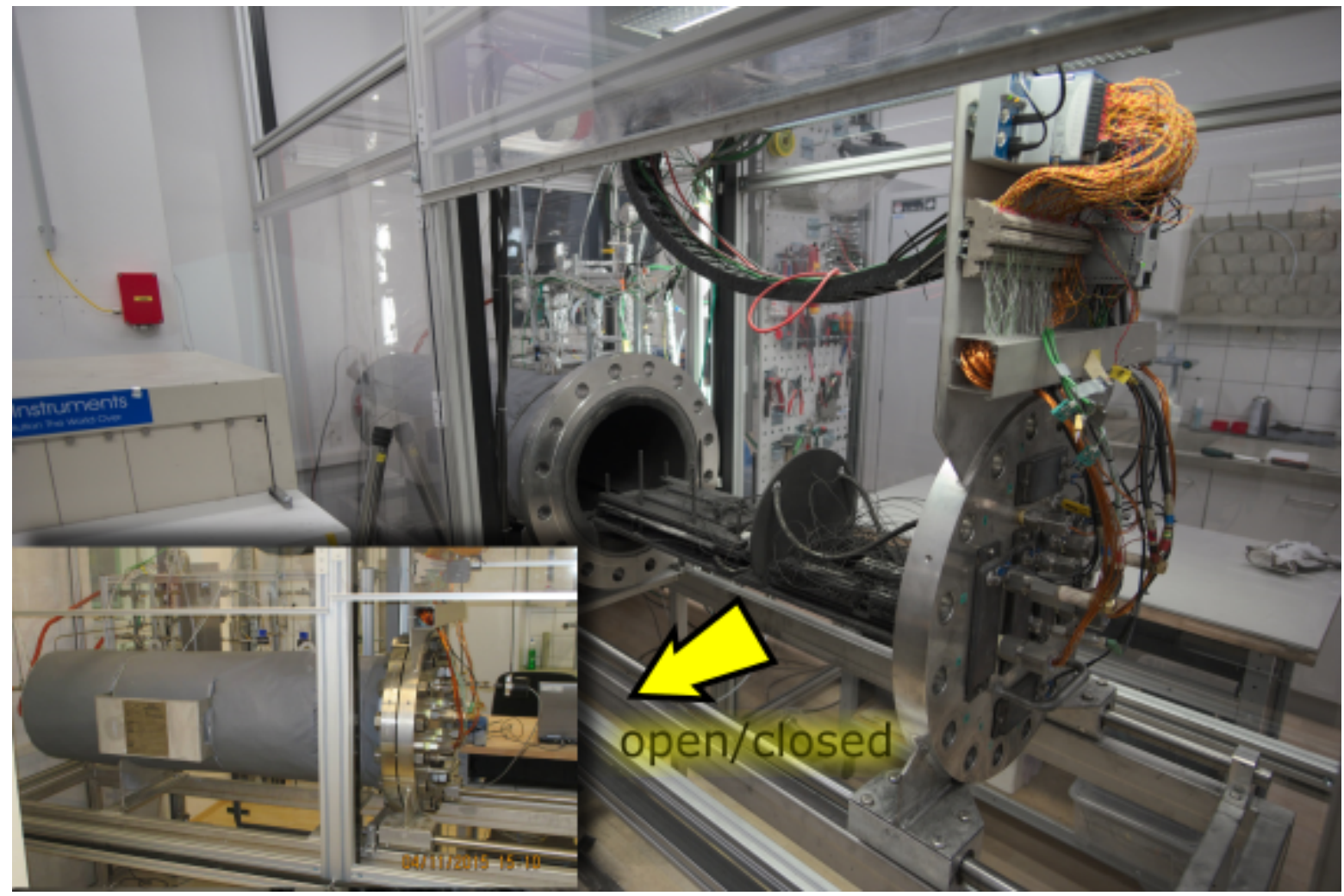

Figure 2. Sealable reactor for thermal runaway experiments 


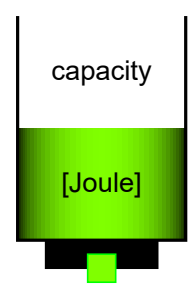

Figure 3. Icon of the EnergyStorage model

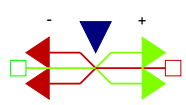

Figure 4. Icon of the VariableEnergy2HeatConversion model

and an energy storage model analogous to a capacitor with a fixed flow rate constant of 1 .

Listing 2. Equation section of the EnergyStorage model equation:

$E=$ port. $E$;

$\operatorname{der}(E)=$ port.P;

All attempts at inhibiting energy outflow when the energy reached 0 , within the model, were unsuccessful. Therefore this was handled in the model converting the energy from the storage to heat, at the rate given by the real input $u$.

Listing 3. Equation section of the VariableEnergy2HeatConversion model

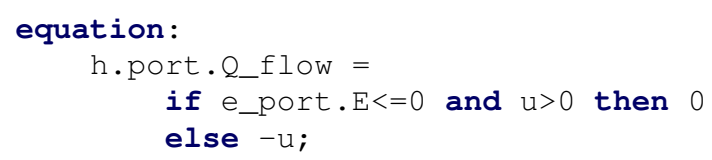

\subsection{Thermal Runaway Model}

With the models for storing and converting energy it is easy to translate Equation 9 into the ChemicalHeatGeneration Modelica model as Figure 5 shows. The temperatureSensor feeds the thermal_port temperature into the HeatEmissionFunction, which corresponds to the linear approximation in Equation 6. Then power_a takes the result to the $10^{\text {th }}$ power, resulting in the conversion rate for conversion2heat and thus draining the energyStorage and releasing heat. The library also features a second heat release model, extending the one explained here. In the extended model a boolean output was added to indicate when the output of the HeatEmissionFunction is greater than a certain threshold. This was done to have a reliable way of telling other models in the system, that the cell has transited into TR.

\subsection{Cell Model}

Figure 6 shows the cell model, to ease implementation it is based on a prismatic cell. From top to bottom it's 3 sub-models are:

1. The electric cell model

2. The heat release or thermal-runaway model

\section{The heat distribution model}

Each of these are replaceable to allow the user to select different model options as they see fit. However, as our focus lay on TR propagation, the included options are limited. There are two electric models in the library, consisting of a voltage source and a resistor. In one the resistor does not generate heat and in the other the resistor generates joule heat. The heat distribution models consist of heat capacitors and heat resistances modelling the cells thermal mass and allowing for either 1D (shown in Figure 10) or pseudo 2D (shown in Figure 8) heat exchange with other models. Furthermore the heat resistances have been adapted to switch to a lower resistance once the cell transits into TR. This was done to accelerate TR propagation, as the venting of hot gas, which plays a major role in TR propagation, has not been implemented yet (Srinivasan et al. 2020). The TR models have been discussed in the previous section. The records in the cell model serve the purpose of providing parameters for the models. These will be discussed later on. The internal heat-port labelled CellTemperature allows to manually read out the cell temperature more conveniently. This also allows to have an external model heat or cool the cell from the inside, if desired. At this point we want to mention that some simple models for heating and cooling are implemented in the library. The heaters are for the most part modelled after their real life counterpart we use in our laboratory, but in a simplistic way. These are used to let the cell transit into TR. The methods of cooling were implemented as a proof of concept but without having a foundation in reality. This was done to gain the experience necessary to implement a thorough model in the future. For the purpose of this paper we will not explain these models in more depth, the interested reader is advised to look at the mentioned models in the package.

\subsection{Module and Pack Models}

Once the cell model is completed and allows for thermal connection in multiple directions, modelling batterymodules (Figure 7) and packs becomes a matter of drawing connections. Figure 8 and Figure 9 show how a module is made from connected cells and how a battery pack is made from several connected modules.

\subsection{Parameter Records}

As mentioned in subsection 3.3, we use records to provide parameters for the models in the package. This provides two advantages. First it reduces the chances of parameterisation errors, as a parameter set for any model has to be entered just once. This also makes correcting such errors easier as there is only one spot where to look. Second, parameters can be changed with a few clicks for many instances of a model at the same time. How powerful these advantages are is evident when looking at the module and pack models in subsection 3.4. In the battery pack are 30 instances of the same module, in each module 12 instances of the same cell and each cell needs 20 parameters. That 


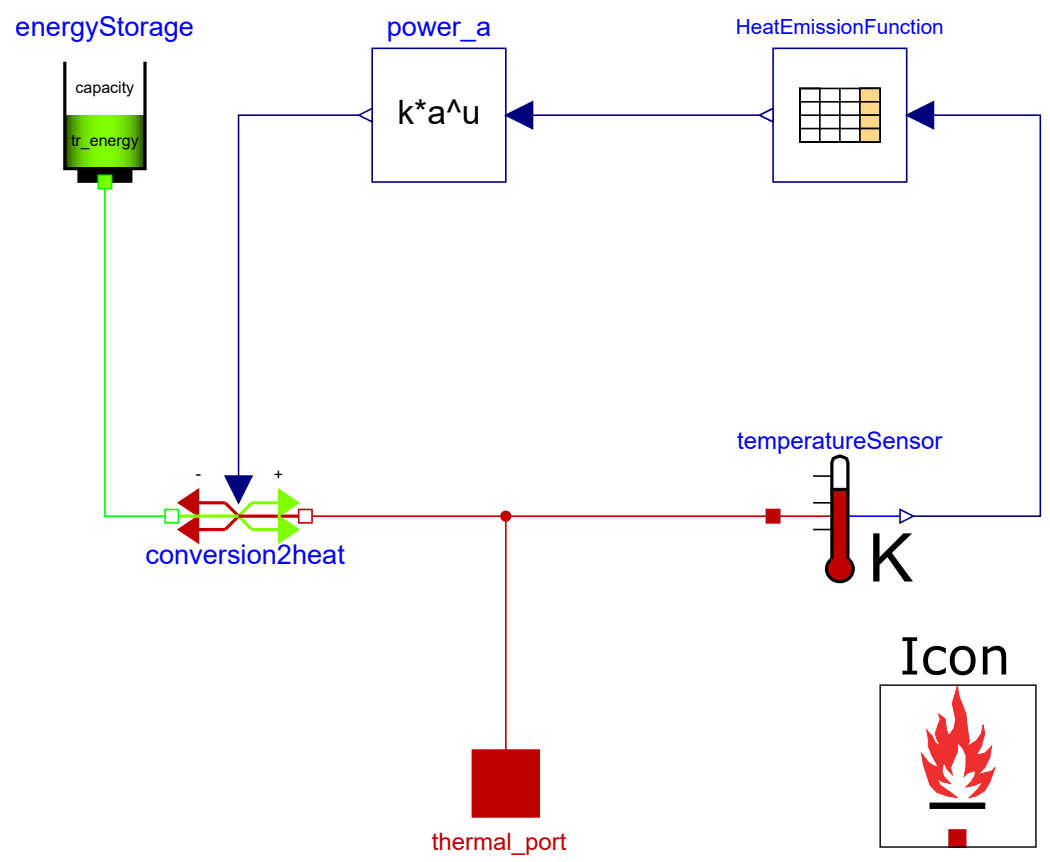

Figure 5. Diagram view and Icon of the ChemicalHeatGeneration model

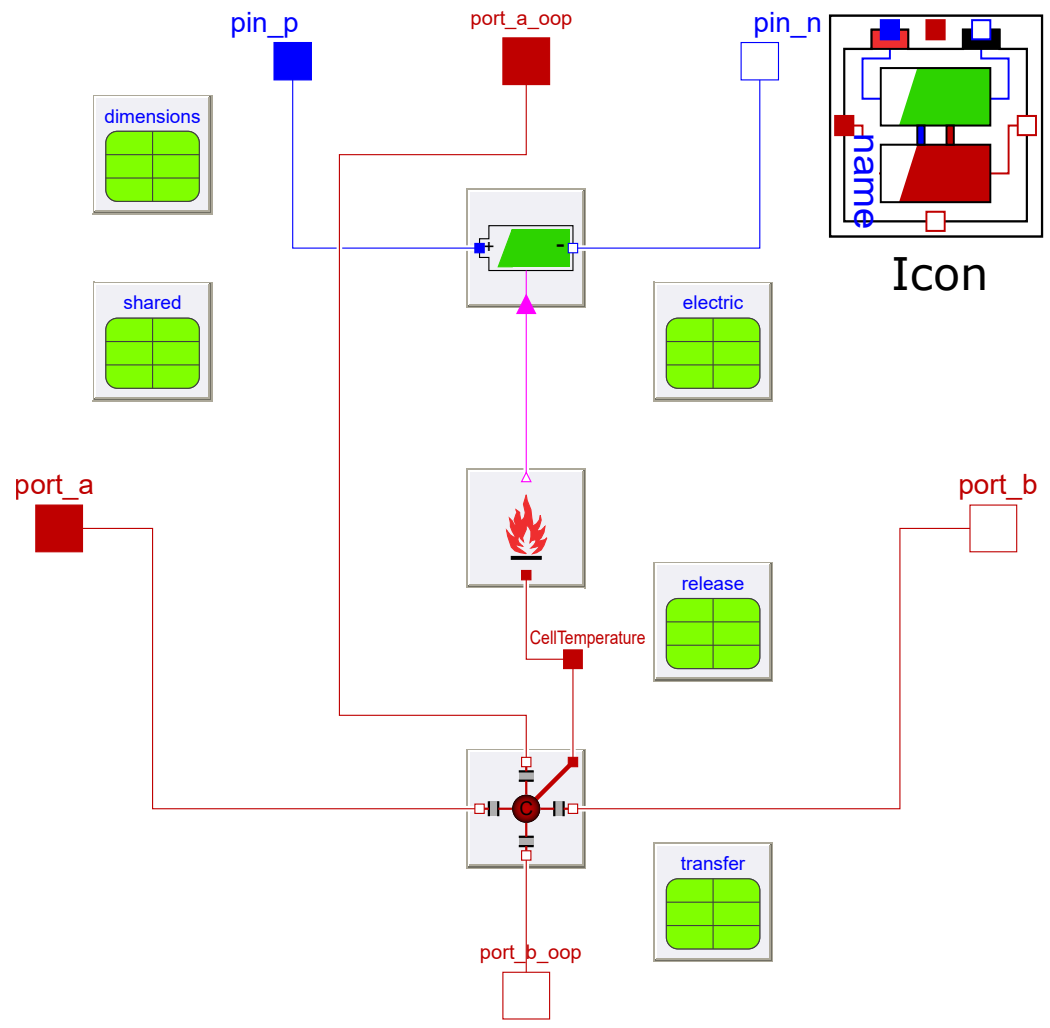

Figure 6. Diagram view and Icon of the cell model. The thermal connectors port_a and port_b transfer heat parallel to the electrode stack (in plane), while port_a_oop and port_b_oop transfer heat orthogonal to the stack (out-of-plane) 


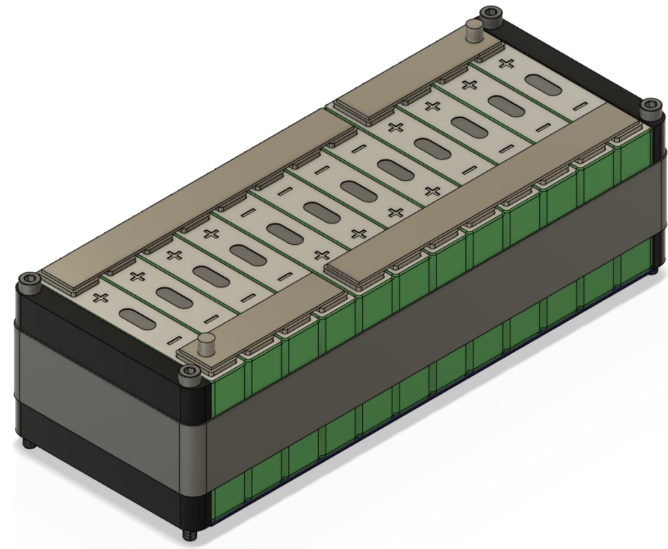

Figure 7. Sketch of a module consisting of $12 \mathrm{Li}$-ion cells with prismatic casing stacked in a row and electrically connected in $4 \mathrm{p} 3 \mathrm{~s}$ configuration (4 parallel, 3 series).

means manually setting the parameters of all the cells requires 7200 inputs.

To further aid the user, the records are defined in groups fitted to the cell sub-model they intend to provide parameters for. Parameters shared between two or more submodels are grouped in a separate record definition. All the records needed to fully parametrise a cell are embedded in another record definition. Each record definition has some data-filled records stored within the library.

\section{Examples}

Now we want to demonstrate the capability of the library with three examples, from single cell to battery pack level. For each example the simulation setup is described and the simulation result is shown. How and to which extend these results can be interpreted will be addressed in section 5 .

\subsection{Single Cell}

For our first example we want to show, that our model correctly captures the thermal behaviour of a LIB. Therefore this example features a single cell with one sided heating as Figure 10 shows. The second heater is turned off and is just there so that the released heat has somewhere to go, which makes the temperature curve look a bit more natural. Figure 11 shows the result, which when compared to measurement data indicates that the dynamics of TR have been captured correctly by our model.

\subsection{Module}

With the second example we want to highlight the scalability of our model. In essence the setup remains the same, but instead of a single cell a module of 12 cells is heated from one side (Figure 12). This setup models the module in a insulated, robust and undamaged casing (no heat exchange with the casing) in an atmosphere devoid of oxygen where no air may enter. The resulting cell temperatures, as shown in Figure 13, display TR propagation through the entire module. This reassures that the developed TR model is capable of portraying this kind of behaviour.

\subsection{Pack}

The last example in this paper concerns the simulation of an entire battery pack, or traction battery, capable of powering an electric car. This was done to show that the model is applicable on this scale with reasonable computation time. Figure 9 shows the layout of the pack, consisting of an array of $3 \times 10$ modules and TR is initiated by heating the cell in the lower left corner. Each module contains 12 cells for a total of 360 cells. Same as for the module example we consider the pack to be encased and the atmosphere devoid of oxygen. The amount of cells simulated make it hard to grasp the full picture just by line-plotting temperature versus time. Therefore we used a MATLAB script to turn the simulation data into a video. This video depicts all of the cells positions and temperatures by colour throughout the entire duration of the simulation. Figure 14 shows some of the videos frames depicting the propagation of the TR throughout the pack.

\section{Discussion}

To summarise: The BatterySafety library features a TR model for LIB based on the simple-tracing model discussed in section 2 . This TR model is then coupled with a simple electrical model and a heat conduction based heat transport model to form a cell model. Cell models are connected to form a module model and module models are connected to form a model of a battery pack. Heater models are used to heat an initial cell until transiting into TR. The cooling models included in the library are at an early stage of development and should only be seen as a proof of concept. The combination of the simple-tracing model with Modelica allows for fast simulations of TR propagation on the scale of a battery pack.

However additional work needs to be done to ensure the validity of the results. At the current state we only have data from cells in a constant volume $\mathrm{N}_{2}$ atmosphere with the same initial pressure. This data is valid to use for simulations considering an intact case of a module or battery pack, that can withstand TR at constant volume. We believe this to be a realistic scenario in the case of a non-crash related thermal event happening in a battery pack. We have found no literature how in $\mathrm{N}_{2}$ atmosphere different initial pressures affect thermal behaviour, either at constant volume or constant pressure. There is literature on the effects of different initial pressures in constant pressure air environment affecting heat released during TR, with higher heat release at higher pressure(Xie et al. 2020; Chen et al. 2019). However we believe this can not be extrapolated to the case of $\mathrm{N}_{2}$ atmosphere, as we see the cause of the higher heat release at higher pressure in the higher availability of oxygen. The effects of different initial pressures in $\mathrm{N}_{2}$ atmosphere on the heat released during TR in constant pressure and constant volume environments is a topic for research in the near future. 


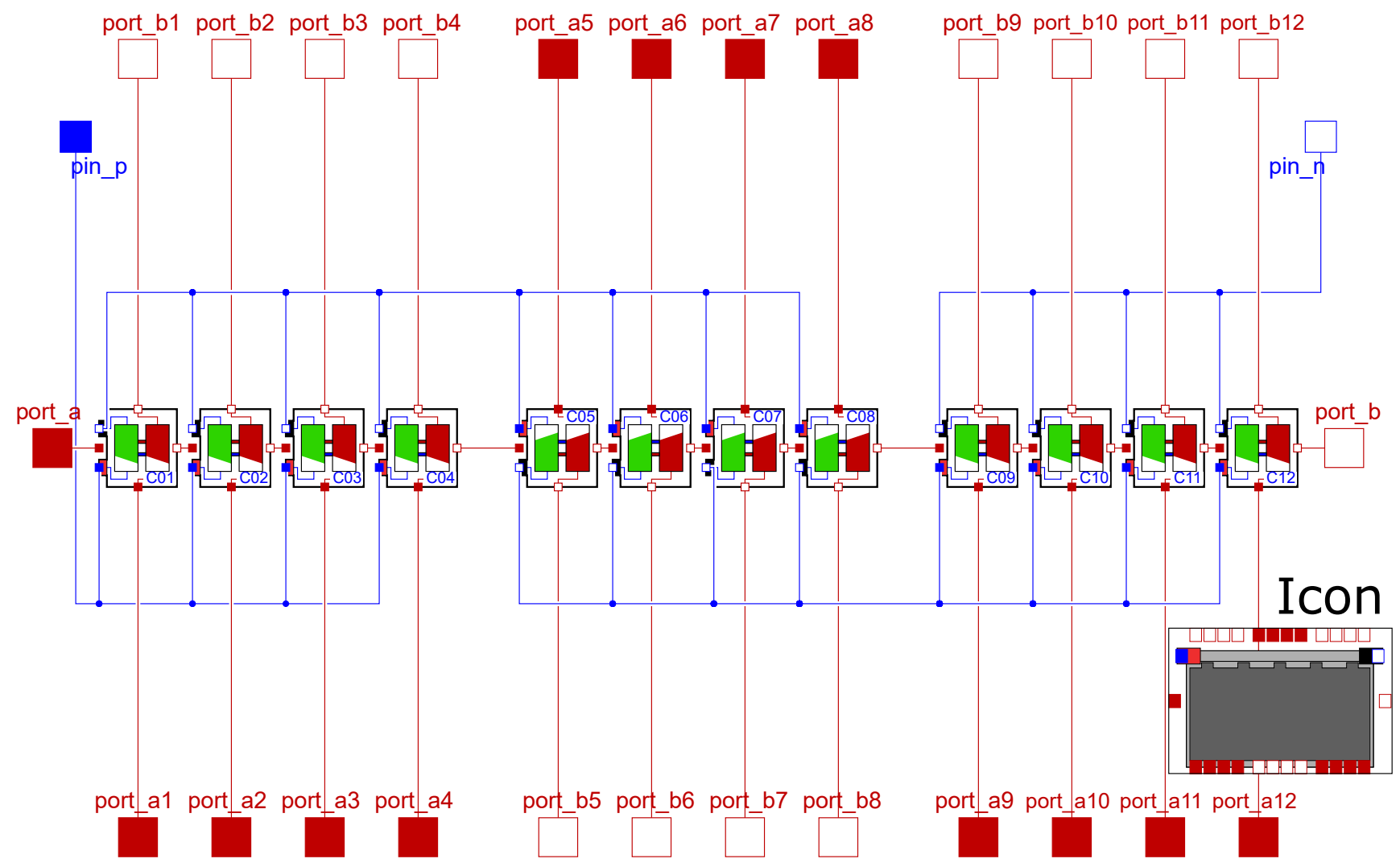

Figure 8. Diagram view of a 12 cell module. The 12 cells $(\mathrm{C} 01 \ldots \mathrm{C} 12)$ are geometrically stacked in a row. Each 4 cells are electrically connected in parallel and the 3 electric cluster are electrically connected in series (4p3s configuration).

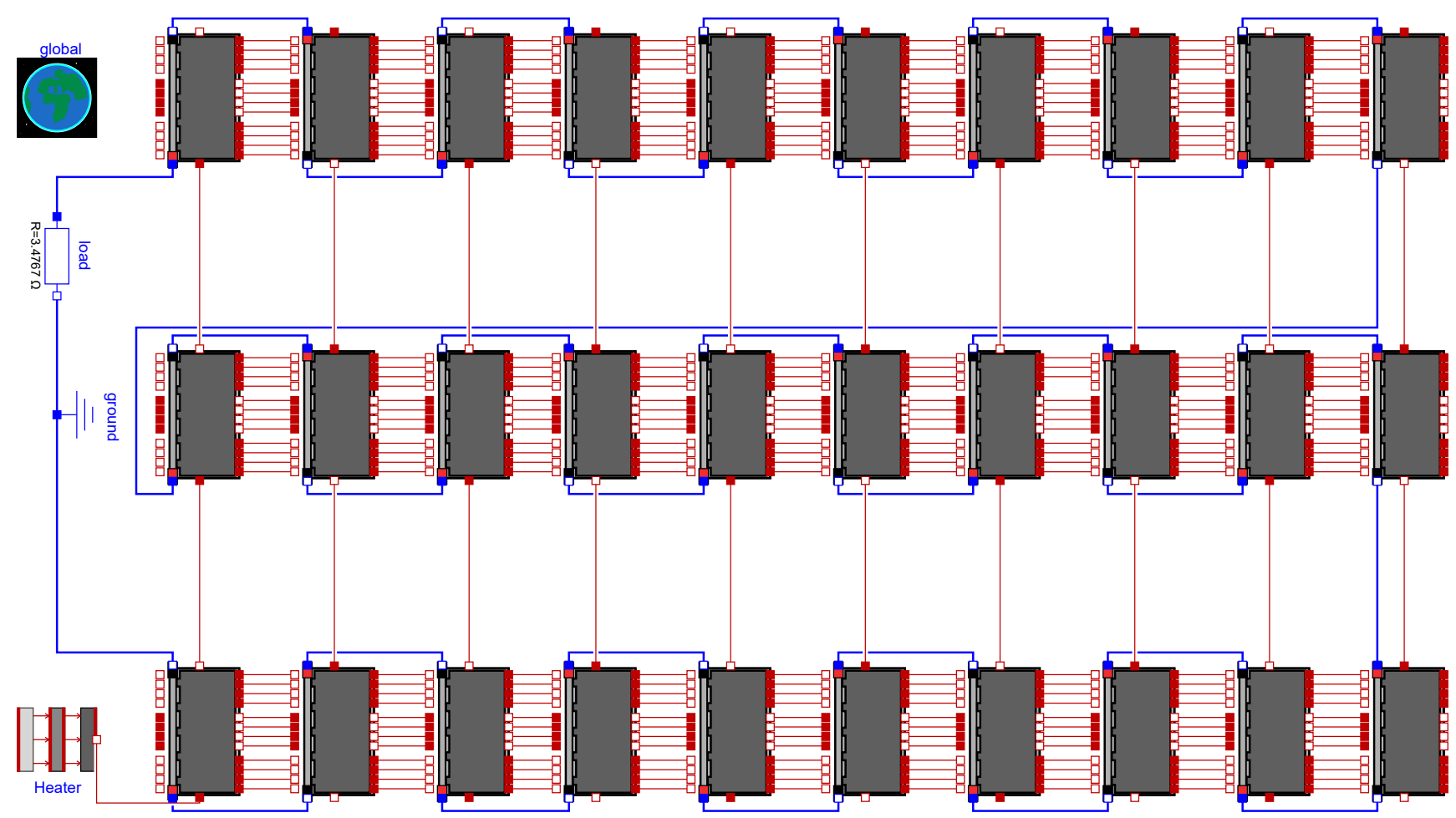

Figure 9. Diagram view of a battery pack. The pack consists of 30 modules which are geometrically arranged in a $3 \times 10$ pattern. All modules are electrically connected in series. The electric loop is closed by a resistor which represents the main electric motor in an electric car. 

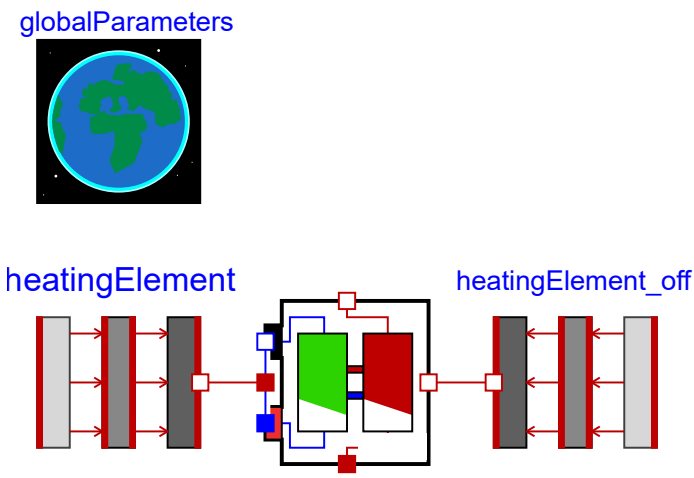

Figure 10. Diagram view of the single cell example. The cell is sandwiched between two heater plates.

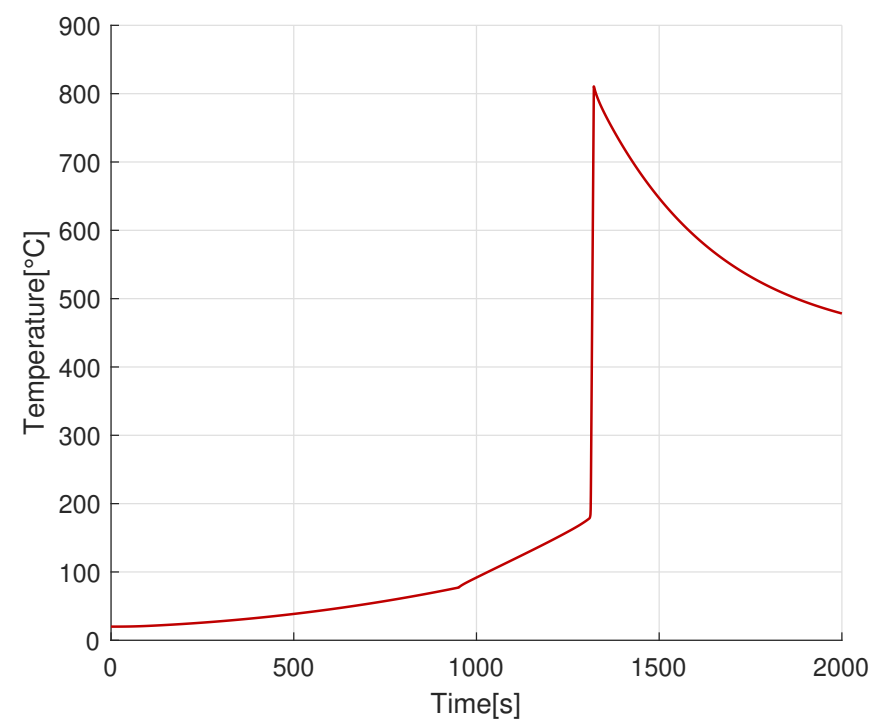

Figure 11. Temperature of a simulated cell. The cell is heated by one of the heater plates until - at $1300 \mathrm{~s}$ - the cell transits into TR. During the TR the cell reaches a temperature above $700^{\circ} \mathrm{C}$. After the TR the cell starts to cool down slowly.

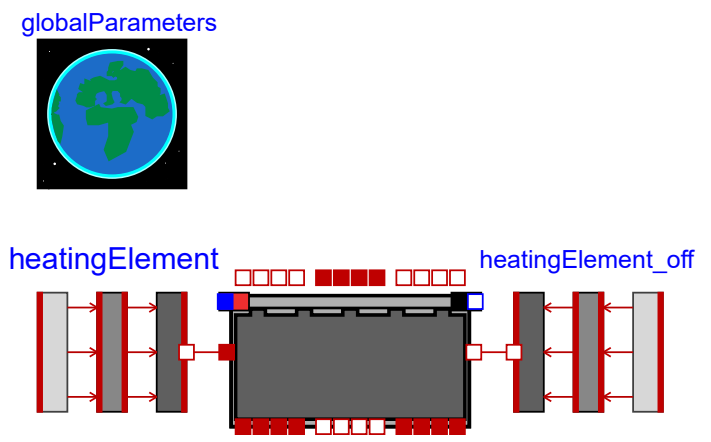

Figure 12. Diagram view of module example. Here the module is sandwiched between two heater plates.
We are aware that a major factor contributing to propagation speed is likely to be the release of high velocity hot gas (venting) as a cell transits into TR (Srinivasan et al. 2020), which is not considered in the library yet. To counteract this fact the cell models feature variable heat resistors, which lower their thermal resistance once the cell transits into TR. This is easy to implement and computationally less expensive compared to adding models for gas release. Whether this approach leads to reliable and generalizable simulation results, or if the implementation of gas release is absolutely necessary has to be determined. Therefore a comparison of these two approaches, as as well as verifying the model will be the topic of a paper in the near future. Further topics worth investigating may be:

- Implementing and researching the effects of active cooling measures and other thermal safety features.

- Implementing interactions between a cells electric model and the energy storage for SOC dependent heat release and more accurate electric behaviour.

- Short-circuits and electric arching between modules, which can be caused by conductive particles released by cells during TR.

- Combustion of vent gases with air, when air enters the battery pack.

- Coupling the model with a FEM cell level simulation to more accurately capture heat distribution in the cell where TR first is initiated.

- Integrating the model in a complete vehicle cosimulation

- Finding a way to estimate the temperature rate curve without the data from a TR experiment

- Finding a relationship between known cell parameters and TR onset temperature.

\section{Conclusion}

We introduced the simple-tracing model for TR in LIB and implemented it in a Modelica package called BatterySafety alongside several other models, to study and further our understanding in TR propagation. Through the discussed examples we have shown the applicability of the developed package to realistic use-cases. The set of such cases is small for now but will continue to grow as research progresses and the package is updated.

The package is easy to use and the models computationally inexpensive even on the scale of a battery pack found in electric vehicles. This allows even inexperienced users to use our TR model to simulate a range of TR propagation simulations with little effort and time and will be available to everyone under the Modelica License 2. To our knowledge the capability to simulate TR propagation through 

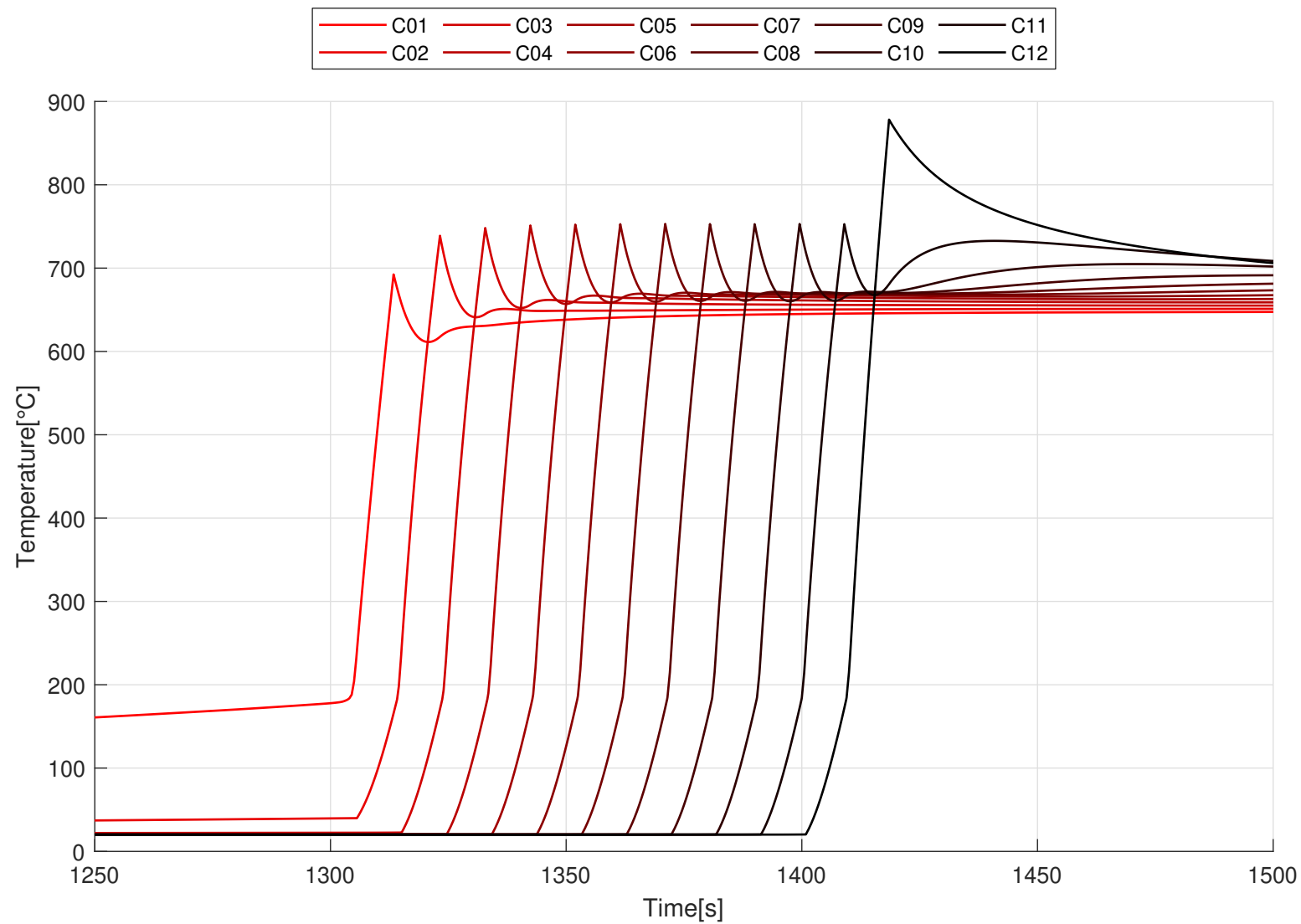

Figure 13. Temperature curves of a simulated module during TR propagation. Each curve belongs to one cell. The TR propagation starts with cell $\mathrm{C} 01$ and finishes with the cell C12.

Time $/ \mathrm{s}=1407$

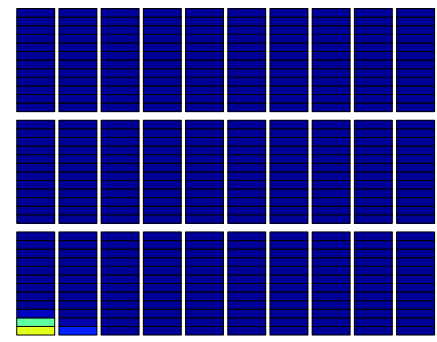

Time $/ \mathrm{s}=1547$

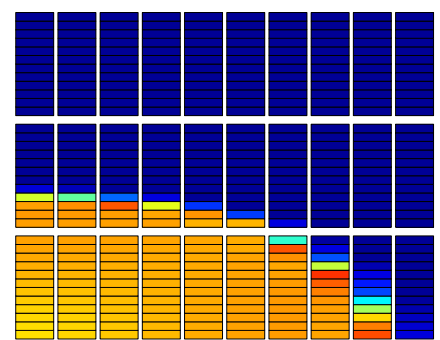

Time $/ \mathrm{s}=1460$

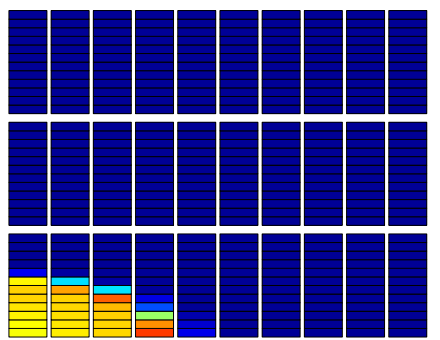

Time / $\mathrm{s}=1619$

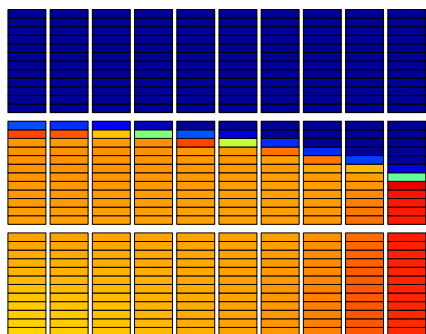

Time $/ \mathrm{s}=1510$

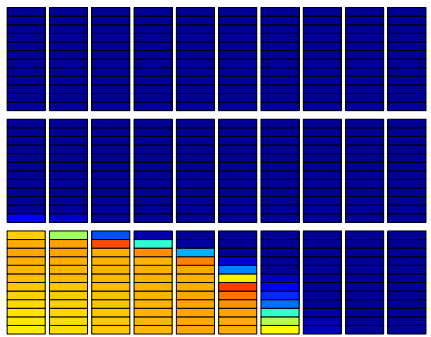

Time / $\mathbf{s}=1661$

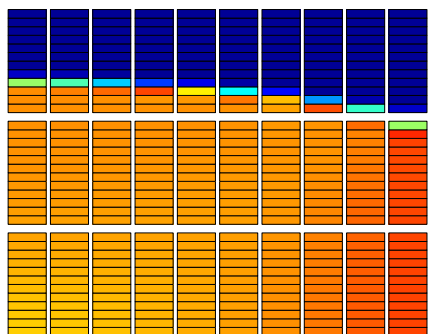

Figure 14. Visualising the TR propagation in a simulated battery pack. Each small rectangle represents a cell. Each stack of cells represents a module. The TR starts in the bottom left cell. With time the TR propagates from cell to cell inside the modules and from module to module. 
a battery pack in a reasonable time is novel. Complex 3D simulations with FEM could take weeks or months for such a simulation in contrast to the 1-4 hours our model takes. Looking back at the requirements on the model from Feng, Lu, et al. (2016) in section 1 we can conclude that with the work done so far, we are about halfway to realising viable TR propagation simulations on a battery pack level.

We consider this paper and the BatterySafety library as the foundation to enable fast, low-skill, virtual prototyping for LIB in the future. We hope that the scientific community will take notice of the value Modelica offers to LIB research and aid us in improving upon our work. We believe this package can help to design safer battery packs in the future.

\section{Acknowledgements}

This publication was written at Virtual Vehicle Research $\mathrm{GmbH}$ in Graz. The authors would like to acknowledge the financial support within the COMET K2-Competence Center for Excellent Technologies Program of the Federal Ministry for Transport, Innovation and Technology (BMVIT), the Federal Ministry for Digital and Economic Affairs (BMDW), the Austrian Research Promotion Agency (FFG), the Province of Styria and the Styrian Business Promotion Agency (SFG).

\section{Conflicts of interest}

The authors declare no conflicts of interest.

\section{References}

Chen, Mingyi et al. (2019). "Environmental pressure effects on thermal runaway and fire behaviors of lithium-ion battery with different cathodes and state of charge". In: Process Safety and Environmental Protection 130, pp. 250-256. ISSN: 0957-5820. DOI: https://doi.org/10.1016/j.psep.2019.08.023.

Essl, Christiane, Andrey W. Golubkov, et al. (2020). "Comprehensive Hazard Analysis of Failing Automotive Lithium-Ion Batteries in Overtemperature Experiments". In: Batteries 6.2. ISSN: 2313-0105. DOI: 10.3390/batteries6020030.

Essl, Christiane, AW Golubkov, and Anton Fuchs (2020). "Comparing Different Thermal Runaway Triggers for Two Automotive Lithium-Ion Battery Cell Types". In: Journal of the Electrochemical Society 167.13, p. 130542. DOI: 10.1149/ 1945-7111/abbe5a.

Feng, Xuning, Languang Lu, et al. (2016). "A 3D thermal runaway propagation model for a large format lithium ion battery module”. In: Energy 115, pp. 194-208. ISSN: 0360-5442. DOI: 10.1016/j.energy.2016.08.094.

Feng, Xuning, Siqi Zheng, et al. (2019). "Investigating the thermal runaway mechanisms of lithium-ion batteries based on thermal analysis database". In: Applied Energy 246, pp. 5364. ISSN: 0306-2619. DOI: 10.1016/j.apenergy.2019.04.009.

Golubkov, Andrey W. et al. (2018). "Thermal runaway of large automotive Li-ion batteries". In: RSC Advances 8.70, pp. 40172-40186. ISSN: 20462069. DOI: 10 . 1039 / C8RA06458J.
Srinivasan, Rengaswamy et al. (2020-02). "Preventing Cell-toCell Propagation of Thermal Runaway in Lithium-Ion Batteries". In: Journal of The Electrochemical Society 167.2, p. 020559. DOI: 10.1149/1945-7111/ab6ffo.

Xie, Song et al. (2020). "Influence of cycling aging and ambient pressure on the thermal safety features of lithium-ion battery". In: Journal of Power Sources 448, p. 227425. ISSN: 0378-7753. DOI: https://doi.org/10.1016/j.jpowsour.2019. 227425 . 